\title{
Age-Related Changes in Sensorimotor Temporal Binding
}

\author{
Tiziana Vercillo*, Carlos Carrasco and Fang Jiang \\ Department of Psychology, University of Nevada, Reno, NV, United States
}

The causal relationship between a voluntary movement and a sensory event is crucial for experiencing agency. Sensory events must occur within a certain delay from a voluntary movement to be perceived as self-generated. Therefore, temporal sensitivity, i.e., the ability to discriminate temporal asynchronies between motor and sensory events, is important for sensorimotor binding. Moreover, differences in the physical propagation of external stimuli can sometimes challenge sensorimotor binding, generating illusory asynchrony. To overcome this problem, the brain adjusts the perceptual timing of sensory and motor events. This mechanism, named sensorimotor recalibration, helps keeping causality judgments accurate. As humans age, the broad decline in sensory and motor processing may reduce temporal sensitivity, and compromise sensorimotor recalibration. In the current study, we investigated the effect of aging on sensorimotor temporal binding by measuring changes in both temporal sensitivity and recalibration. Young and elderly adults were exposed to a prolonged physical delay between a voluntary movement (a keypress) and its perceptual consequence (a visual stimulus). Before and after this exposure, participants performed a sensorimotor temporal order judgment (TOJ) task. As expected, elderly adults showed reduced sensorimotor recalibration and sensitivity as

Edited by: Juliana Yordanova, Institute of Neurobiology (BAS), Bulgaria

Reviewed by: Giorgio Innocenti, Karolinska Institute (KI), Sweden Giuliana Lucci, IRCCS Santa Lucia of Rome, Italy

*Correspondence:

Tiziana Vercillo tiz.vercillo@gmail.com

Received: 14 July 2017 Accepted: 29 September 2017 Published: 12 October 2017

Citation: Vercillo T, Carrasco $C$ and Jiang $F$ (2017) Age-Related Changes in Sensorimotor Temporal Binding.

Front. Hum. Neurosci. 11:500. doi: 10.3389/fnhum.2017.00500 compared to young adults, suggesting that aging affects sensorimotor temporal binding.

Keywords: sensorimotor, ageing (aging), recalibration, adaptation, temporal sensitivity

\section{INTRODUCTION}

Agency is central to human life as it embraces the conscious experience of changing the external world through behavior. Such mental phenomenon is inherently associated with causality judgments and sensorimotor temporal binding (David et al., 2008). Whether it is determining if a twig snapping is caused by one's own footstep or by the movement of a predator, or learning to play a video game, the temporal binding of actions and sensory events is an integral part in defining the sense of agency (Haggard and Chambon, 2012).

Causality assessments require the fine ability to discriminate the temporal order between motor and sensory events, i.e., sensorimotor temporal sensitivity. A common notion of causality is that only sensory events that directly follow the onset of a voluntary movement are considered consequences of our own actions rather than the effects of external agents (Blakemore et al., 1999; Moore et al., 2009). Therefore, motor and sensory events that occur within a certain temporal window are usually bound together. On the other side, unrelated information that violate the notion of causality are segregated (Blakemore et al., 1999; Moore et al., 2009; Moore and Fletcher, 2012). 
In this respect, the perceived temporal relationship between motor and sensory events represents a major factor for causality judgments and sensorimotor binding.

The subjective experience of agency is regulated by the synergic activity of the motor and the sensory systems that process and integrate information from action planning and execution with sensory inputs (David et al., 2008; Moore and Fletcher, 2012). Sensorimotor binding yet must account for differences in the temporal processing within sensory pathways and between sensory and motor pathways (Eagleman et al., 2005). For instance, while motor and proprioceptive inputs are generated and processed within the body, visual and auditory inputs typically travel through the environment to reach the brain. The mechanism of sensorimotor recalibration reduces misleading delays and, in accordance with prior knowledge and/or recent sensory history, regulates the perceptual timing of motor and sensory events keeping causality judgments accurate (Eagleman and Holcombe, 2002; Stetson et al., 2006; Eagleman, 2008; Heron et al., 2009; Sugano et al., 2010; Keetels and Vroomen, 2012). Even a brief exposure to a temporal delay between a simple motor act and its sensory consequences can trigger sensorimotor recalibration (Stetson et al., 2006). The physical delay is wiped out, moving the two events close together.

Sensorimotor abilities are immature in children, develop throughout adolescence and reach a plateau in early adulthood (Vercillo et al., 2014). However, the aging process causes gradual losses to the sensory (Warren et al., 1978; Burton et al., 1993; Spear, 1993; Vesco et al., 1993; Spear et al., 1994; Sturr et al., 1997; Chisolm et al., 2003; Clinard et al., 2010) and the motor system (Erim et al., 1999; Seidler et al., 2010). Even older adults who are free from cognitive impairment still show signs of sensory and motor decline and poor spatial sensorimotor adaptation (Bock and Schneider, 2002; Bock, 2005). More recent works also suggest that temporal sensitivity declines with age, as elderly individuals showed a great difficulty in detecting audiovisual asynchrony (Chan et al., 2014; Stevenson et al., 2017). Moreover, there seems to be indications of a slowing of the internal clock with age indicated by results from unpaced tapping tasks (Turgeon et al., 2011; Turgeon and Wing, 2012). Whether the decline in sensory and motor functions as well as in the sensitivity of temporal perception affects sensorimotor temporal binding is nevertheless still unclear.

In the current study, we investigated sensorimotor temporal binding in young and elderly individuals by measuring: (1) sensorimotor temporal sensitivity, as the ability to discriminate the temporal relationship between motor and sensory events; (2) sensorimotor recalibration, as the ability to adjust the timing of motor and sensory events after adaptation to a sensorimotor asynchrony. Participants were tested in a task requiring the discrimination of the temporal order between a motor and a sensory event. The task was performed before and after the exposure to a temporal delay between their own action and the subsequent sensory consequence. As predicted, we found that aging is associated with a decline in sensorimotor temporal binding.

\section{MATERIALS AND METHODS}

Ten elderly participants (mean age: $68 \pm 0.57,6$ females and 4 males) and 11 young adults (mean age: $31 \pm 3$ years, 7 females and 4 males) participated in this study. All participants were right handed and had normal or corrected to normal vision. Two elderly participants required the use of hearing aids. All the elderly participants reported no cognitive impairment or neurodegenerative diseases. Younger participants were recruited from the University of Nevada, Reno while elderly individuals were recruited from the Reno (NV) area. All participants read and signed an informed consent before the experiment. The Institutional Review Board at the University of Nevada, Reno approved all experimental protocols.

Experimental procedures and stimuli were adapted from Vercillo et al. (2014). The visual stimulus consisted of a white circle measuring $6^{\circ}$ in diameter that was briefly flashed on a gray background for $30 \mathrm{~ms}$. The stimulus was presented on a Display ++ LCD monitor (Cambridge Research System). Motor actions consisted in button presses and were recorded via a CB6 response box that interfaced with Bits\#. To cover the sound produced by the button press, participants listened to $65 \mathrm{~dB}$ white noise that was delivered through headphones.

During the experiment, participants sat in a dark room at $57 \mathrm{~cm}$ from the screen. Sensorimotor synchronization and temporal precision were assessed with a temporal order judgement (TOJ) task. At the beginning of each trial of the TOJ task, a black fixation cross appeared in the center of the screen. After $2 \mathrm{~s}$, the fixation cross disappeared and participants were required to perform a voluntary action by pressing the button on the response box as fast as possible. Participants were trained to perform fast and precise movements. The visual stimulus (a white circle) was displayed at a variable temporal interval from the disappearance of the fixation cross, so that it could have appeared either before or after participants' button press. At the end of each trial, participants verbally reported whether the circle appeared before or after their button press, thus making a TOJ. Verbal responses and reaction times (RTs) were both recorded in Matlab.

Asynchronies between the visual stimulus and the motor action were partially determined by individual average RTs. First, we calculated individual average RTs, and then we utilized these values to determine stimulus latencies. Latencies were calculated from the timing of the disappearance of the fixation cross and were selected to ensure that the visual stimulus was presented either before or after participants' button press. Specifically, we tried to establish the following asynchrony values (Stimulus Onset Asynchrony-SOA): $\pm 100 \mathrm{~ms}, \pm 80 \mathrm{~ms}, \pm 60 \mathrm{~ms}, \pm 40 \mathrm{~ms}$, $\pm 20 \mathrm{~ms}$ and $0 \mathrm{~ms}$, where negative values indicate that the visual stimulus occurred before the motor action, and positive values indicate that the visual stimulus happened after the motor action. Each latency was repeated 10 times in a constant stimuli algorithm. Note that as individual's RTs naturally fluctuated around the average, the effective SOA values diverged from those originally selected values. Using this strategy, we were able to deliver the stimulus as early as $300 \mathrm{~ms}$ before and $300 \mathrm{~ms}$ after the button press. 
At the beginning of the experiment, each participant ran a brief training session of 30 trials to familiarize with the TOJ task. During the training, average RTs were fixed at $250 \mathrm{~ms}$. The learning process was facilitated by the occurrence of a feedback to individual responses at the end of each trial. Following the training session, we measured individual average RTs and set stimulus latencies for the TOJ task.

During adaptation, participants were exposed to a $200 \mathrm{~ms}$ delay between the motor action and the visual feedback. Participants were instructed to fixate the cross in the center of the screen and press a button at their own will receiving a visual feedback with a delay of $200 \mathrm{~ms}$. We randomly presented catch trials with a different visual stimulus (a dark gray circle) and asked participants to count them and verbally report the number at the end of the adaptation phase. Catch trials were selected from a range of $30-50$ by the experimenter with the aim of ensuring participants' attention and preventing short inter-press intervals.

Participants performed two experimental blocks: a baseline block, consisted of 110 TOJ trials and an adaption blocks that consisted of 100 adaptation trials followed by 110 TOJ trials. The baseline block was performed first. After the baseline block, individual average RTs were recalculated and updated for the adaptation block. Figure 1 shows experimental procedures.

For each experimental block, the proportion of trials where the visual stimulus was perceived as happening after the motor action was computed for each effective SOA value and fitted with cumulative Gaussian functions. The two parameters of the Gaussian (mean and standard deviation) were estimated using the maximum likelihood method (Finney, 1947). The mean value represents the point of subjective simultaneity (PSS), while the standard deviation represents the just noticeable difference (JND).

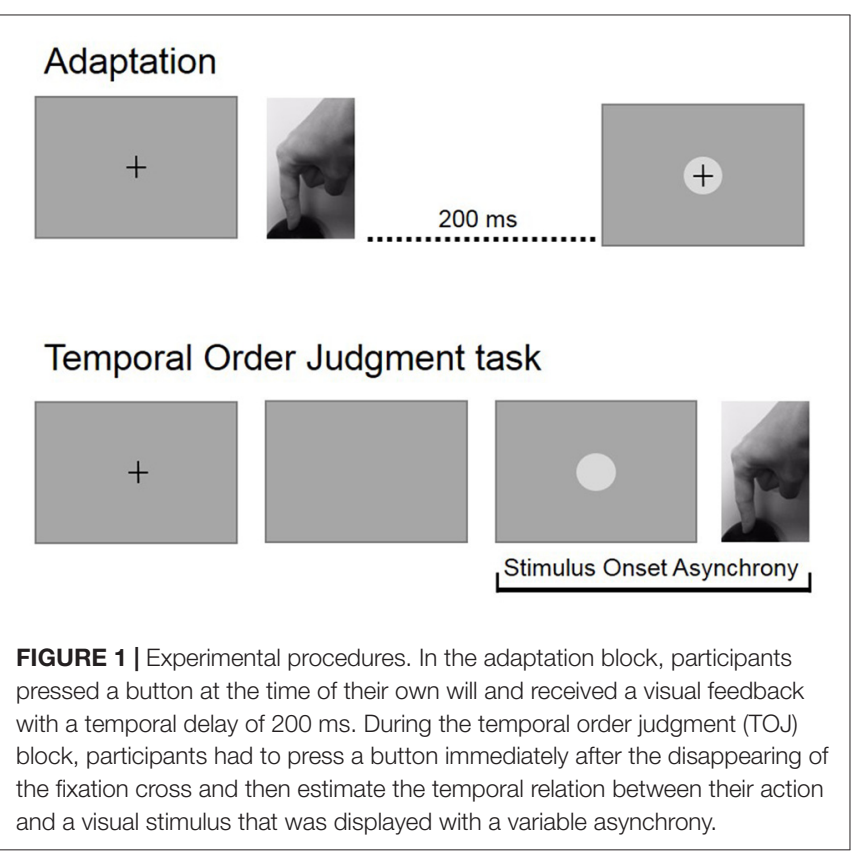

The PSS represents the SOA value at which the delay between the motor and the sensory event is indiscernible for the participant. The PSS represents a non-discrimination point and is defined as the $50 \%$ point of the psychometric function. More in general, the PSS represent the perceived simultaneity between the motor action and the visual stimulus. A PSS equal to 0 indicates that motor and sensory events are perceived as synchronous when they physically occur at the same time. PSS values different from 0 denote a perceptual bias. Specifically, a positive PSS value indicates that the sensory and the motor events have been moved closer in time. In this case, the perceived synchrony befalls when the visual stimulus is delayed. After measuring PSS values in the adaptation and the baseline condition, we assessed the recalibration effect by subtracting these two values. A positive effect denotes sensorimotor recalibration: a temporal compression between the sensory and the motor events.

As an index of sensorimotor temporal sensitivity, we measured the JND, the standard deviation of the function. The JND represent the minimum temporal delay between motor action and visual feedback to produce a JND in temporal perception. Standard errors for the PSS and JND estimates were obtained with a bootstrap procedure (Efron and Tibshirani, 1993). Trials from the training block were excluded from analysis. We also measured RTs during the TOJ task, calculated as the temporal interval between the disappearance of the fixation cross and the button press.

Sensorimotor recalibration was measured as the difference in the PSS between the adaptation and the baseline condition. Values were calculated individually and then averaged across participants for each group.

Data were analyzed with two-tailed $t$-tests, with the Analysis of Variance (ANOVA) and with Linear Regression Analysis using SPSS 22 software (SPSS, Inc., Chicago, IL, USA).

\section{RESULTS}

Differences in sensorimotor temporal binding between the two groups of participants are reported in Figure 2. The left panel

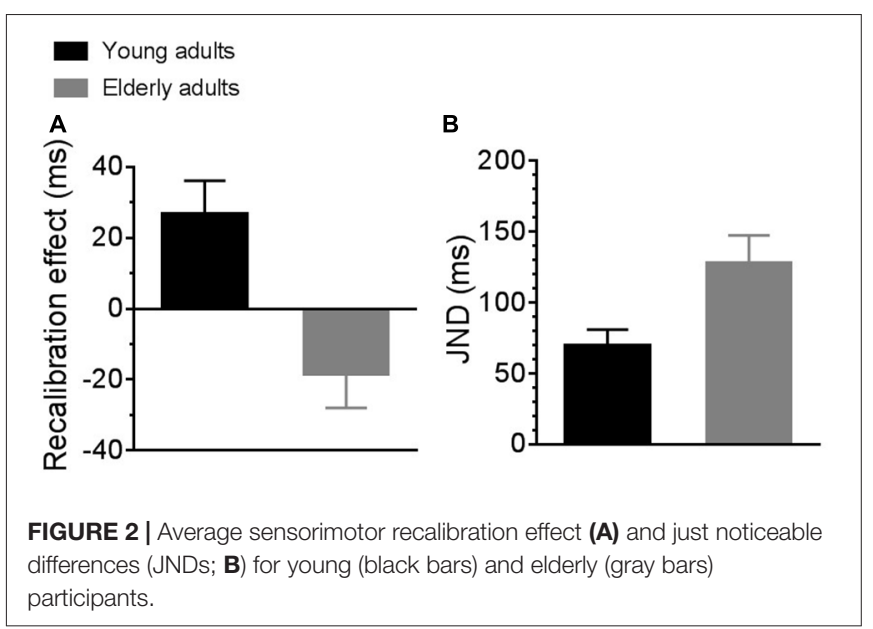


of the figure shows the recalibration effect for young (black bar) and elderly (gray bar) adults. Young adults showed a significantly higher recalibration effect as compared to elderly adults (2-tailed, independent samples $t$-test with Bonferroni correction for multiple comparison; $t_{19}=3.45, p=0.003$ ). In particular, young adults showed a positive recalibration effect greater than 0 (2-tailed, one sample $t$-test with Bonferroni correction for multiple comparison; $t_{10}=2.9, p=0.01$ ) while elderly adults showed a tendency toward a negative effect of recalibration that nevertheless was not significant (2-tailed, one sample $t$-test with Bonferroni correction for multiple comparison; $t_{9}=-1.99$, $p=0.07)$.

The right panel of the figure shows average JNDs for young (black bar) and elderly (gray bar) adults. JNDs for elderly participants were significantly higher than for young participants, suggesting poorer sensorimotor temporal sensitivity in the elderly group. A repeated measure ANOVA (experimental condition $*$ group) confirmed an effect of group $\left(F_{(1,19)}=7.66\right.$, $p=0.01$, partial $\left.\eta^{2}=0.28\right)$ but no effect of experimental condition, as JNDs were similar between baseline and adaptation condition. For this reason, in the figure we reported JNDs averaged across experimental conditions.

Average RTs measured during the TOJ task in the adaptation and the baseline condition for the two groups of participants are shown in Figure 3. A repeated measure ANOVA revealed an interaction between experimental condition and group $\left(F_{(1,19)}=5.14, p=0.03\right.$, partial $\left.\eta^{2}=0.21\right)$ and a significant effect of group $\left(F_{(1,19)}=11.33, p=0.003\right.$, partial $\left.\eta^{2}=0.37\right)$. Overall, elderly individuals (gray symbols and line) reacted slower than young participants (black symbols and line) to the go signal.

\section{- Young adults}

- Elderly adults

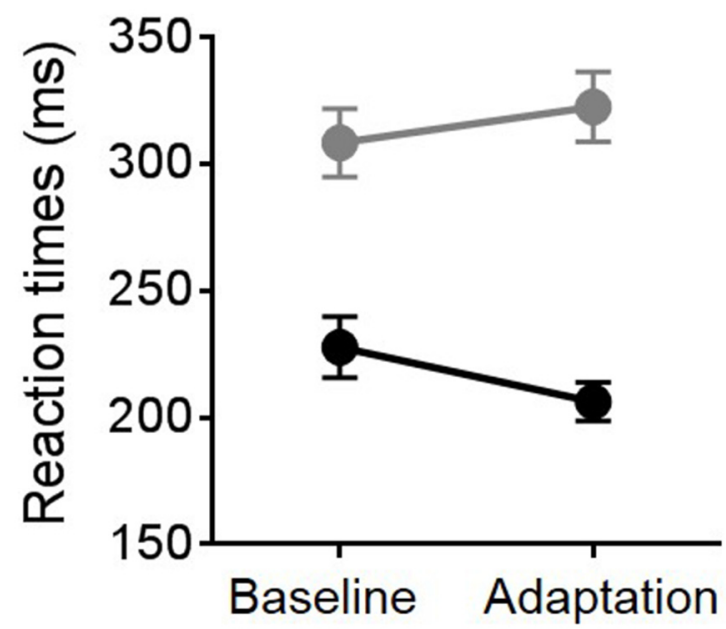

FIGURE 3 | Average reaction times (RTs) for young (black symbols and line) and elderly (gray symbols and line) participants in the baseline and the adaptation condition.
Interestingly, elderly and young adults showed an opposite pattern of responses across the two experimental conditions. While elderly participants slowed down motor responses in the second block (the adaptation block), young participants showed faster RTs during adaptation.

We found a negative correlation between recalibration effect and age $\left(R^{2}=0.23, p=0.02\right)$. Only young adults exhibited a positive recalibration effect. Elderly participants did not compress the temporal interval between action and sensory event, and sometimes even showed an opposite pattern of adaptation. Sensorimotor temporal sensitivity decreased with age, considering that individual JNDs became higher as the age increased $\left(R^{2}=0.38, p<0.005\right)$. Similar to JNDs, RTs substantially increased with age $\left(R^{2}=0.44, p<0.001\right)$. In Figure 4, we reported changes in the recalibration effect, JNDs and RTs associated with age. This figure shows individual data for all the participants $(n=21)$ fitted with a linear regression model.

\section{DISCUSSION}

Agency refers to the experience of controlling our own actions and producing consequences in the outside world (Haggard and Chambon, 2012). In everyday life, we have dealings with a continuous stream of information and sometimes distinguishing sensory events that we produce from those effected by others may be challenging. Sensorimotor binding (the ability of grouping together actions and sensory consequences that are linked by a causal relationship) strongly depends on temporal sensitivity and recalibration. The brain must understand temporal relations between motor and sensory events and adjust these perceptual judgments in case of misleading delays. In the current study, we investigated changes in sensorimotor temporal binding and found a reduced ability of grouping sensory and motor events caused by alterations in temporal sensitivity and sensorimotor recalibration.

It is well documented that aging induces a general slowing of cognitive processing that affects the behavioral performance of elderly adults in several tasks (Cerella, 1985; Birren and Fisher, 1995). However, results from this study together with previous researches on multisensory and sensorimotor integration (Wolpe et al., 2016; Stevenson et al., 2017), support the notion that age-related differences in sensorimotor processing could not be explained by general cognitive slowing. The poor sensorimotor binding might rather be attributable to a decrease in temporal sensitivity and a decline of sensory and motor processing. Indeed, older adults can hardly separate auditory and visual signals in time, as their temporal window of integration widens (Laurienti et al., 2006; Setti et al., 2011; Mozolic et al., 2012; Bedard and Barnett-Cowan, 2016) and these temporal deficits might be related to a reduced sensitivity or acuity in the sensory systems (Schmolesky et al., 2000; Ostroff et al., 2003; Liu and Yan, 2007; Shaffer and Harrison, 2007; Mozolic et al., 2012).

In the course of healthy aging, sensory and motor abilities decline and a linear reduction of the volume in association cortices and white matter becomes apparent (Raz et al., 2005). 

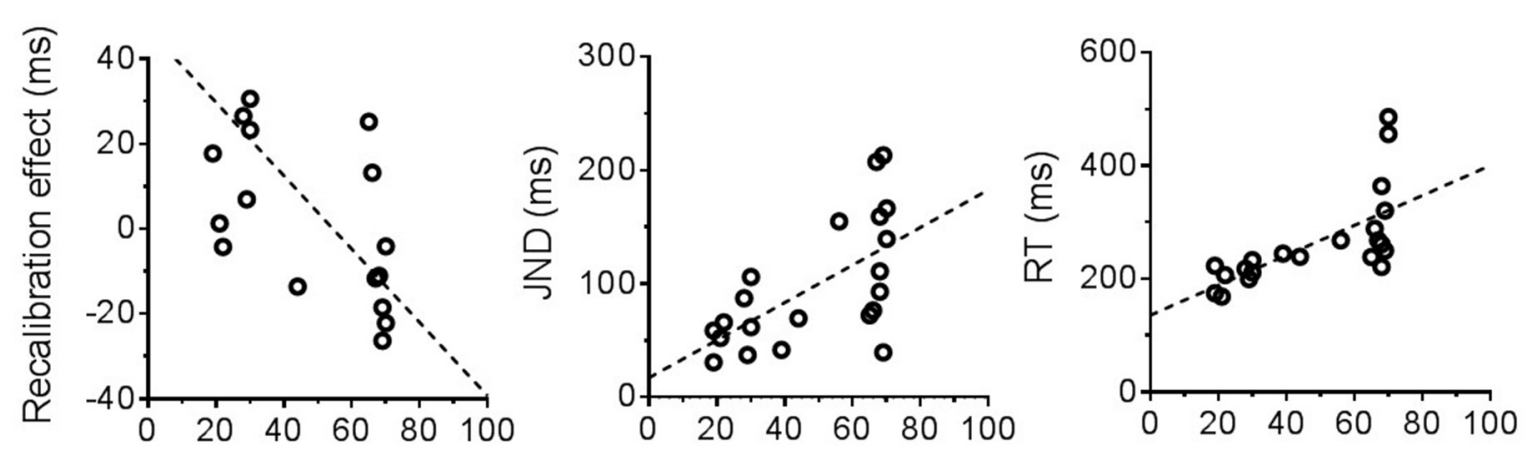

Age (years)

FIGURE 4 | Age-related changes of the recalibration effect, JNDs and RTs.

The combination of sensory information into intentional motor response deteriorates with age generating balance impairments (Maki and McIlroy, 1996) and increasing the risk of falls (Tinetti et al., 1988). Similarly, in our study we found that temporal sensorimotor binding declines with age. Elderly participants experienced troubles in representing causal relationship between motor and sensory events. The lower sensitivity for sensorimotor asynchrony may represent the source of different experiences of agency between young and elderly adults. More importantly, the loss of sensorimotor recalibration with age points out a decrease in the flexibility of sensorimotor binding and a poor adaptability to environmental conditions for the elderly population.

In agreement with our results, a recent study by Wolpe et al. (2016) showed increased sensorimotor attenuation, a reduction in the perceived intensity of sensations when they are induced by a voluntary action, in elderly as compared to young adults. The effect was related to differences between elderly and young adults in the volume and the connectivity of the pre-supplementary motor area with prefrontal and striatal regions. Following the loss of sensory precision, predictive signals generated by voluntary movements may be over-weighted, and sensory information down-weighted. Authors suggested that the increased attenuation in the elderly population might represent a compensatory mechanism to preserve the sense of agency. Indeed, the increased noise within the sensory and the motor pathways might challenge the ability to discriminate between sensory events that are the consequences of our own actions rather than the effects of external agents. Similarly, previous studies found that the ability to integrate multiple sensory signals into a perceptual gestalt declines with age (Stevenson et al., 2017) and that aging impacts on unisensory (Gelfand et al., 1980; Robin and Royer, 1989) and multisensory (Chan et al., 2014) temporal processing. Together, these findings suggest that the binding of sensory and motor input changes across the lifespan, with elderly individuals showing deficit in ordinary tasks such as speech production and perception, and spatial navigation.
Age-related reduction in attentive efficiency (Madden, 2007) and in selective inhibition of irrelevant stimuli (McDowd and Oseas-Kreger, 1991; Chao and Knight, 1997) might also be critical for the lack of sensorimotor recalibration and the poor temporal sensitivity that we found in elderly participants. As described in Figure 3, RTs for elderly participants became slower in the second experimental block, the adaptation block, suggesting fatigue and a possible drop in attentional resources. On the contrary, RTs of young participants became faster, suggesting an automatization of the task. As revealed by a previous study, top-down attention can modulate the ability to adjust temporal judgments based on recent sensory history (Heron et al., 2010) and therefore the involvement of this higher cognitive process deserves further investigations.

Understanding the nature of age-related changes in sensorimotor binding and their connections with a more general decline in cognitive functions is critical for developing interventions aimed to improve the quality of life of elderly individuals. Future researches should clarify the linkage between age-related deficit in sensorimotor and multisensory temporal binding and assess whether they are in some way modulated by impairment in higher cognitive processes.

\section{AUTHOR CONTRIBUTIONS}

TV and FJ: conception and design of study, revising the manuscript critically for important intellectual content. TV and CC: acquisition of data, drafting the manuscript. TV: analysis and/or interpretation of data.

\section{ACKNOWLEDGMENTS}

This research has been supported by Foundation for the National Institutes of Health EY023268 to FJ, EY10834 to Michael Webster, and P20 GM103650. The content is solely the responsibility of the authors and does not necessarily represent the official views of the National Institutes of Health. 


\section{REFERENCES}

Bedard, G., and Barnett-Cowan, M. (2016). Impaired timing of audiovisual events in the elderly. Exp. Brain Res. 234, 331-340. doi: 10.1007/s00221-0154466-7

Birren, J. E., and Fisher, L. M. (1995). Aging and speed of behavior: possible consequences for psychological functioning. Ann. Rev. Psychol. 46, 329-353. doi: 10.1146/annurev.psych.46.1.329

Blakemore, S. J., Frith, C. D., and Wolpert, D. M. (1999). Spatio-temporal prediction modulates the perception of self-produced stimuli. J. Cogn. Neurosci. 11, 551-559. doi: 10.1162/089892999563607

Bock, O. (2005). Components of sensorimotor adaptation in young and elderly subjects. Exp. Brain Res. 160, 259-263. doi: 10.1007/s00221-004-2133-5

Bock, O., and Schneider, S. (2002). Sensorimotor adaptation in young and elderly humans. Neurosci. Biobehav. Rev. 26, 761-767. doi: 10.1016/s01497634(02)00063-5

Burton, K. B., Owsley, C., and Sloane, M. E. (1993). Aging and neural spatial contrast sensitivity: photopic vision. Vision Res. 33, 939-946. doi: 10.1016/0042-6989(93)90077-a

Cerella, J. (1985). Information processing rates in the elderly. Psychol. Bull. 98, 67-83. doi: 10.1037/0033-2909.98.1.67

Chan, Y. M., Pianta, M. J., and McKendrick, A. M. (2014). Older age results in difficulties separating auditory and visual signals in time. J. Vis. 14:13. doi: $10.1167 / 14.11 .13$

Chao, L. L., and Knight, R. T. (1997). Prefrontal deficits in attention and inhibitory control with aging. Cereb. Cortex 7, 63-69. doi: 10.1093/cercor/7.1.63

Chisolm, T. H., Willott, J. F., and Lister, J. J. (2003). The aging auditory system: anatomic and physiologic changes and implications for rehabilitation. Int. J. Audiol. 42, 3-10. doi: 10.3109/14992020309074637

Clinard, C. G., Tremblay, K. L., and Krishnan, A. R. (2010). Aging alters the perception and physiological representation of frequency: evidence from human frequency-following response recordings. Hear. Res. 264, 48-55. doi: 10.1016/j.heares.2009.11.010

David, N., Newen, A., and Vogeley, K. (2008). The "sense of agency" and its underlying cognitive and neural mechanisms. Conscious. Cogn. 17, 523-534. doi: 10.1016/j.concog.2008.03.004

Eagleman, D. M. (2008). Human time perception and its illusions. Curr. Opin. Neurobiol. 18, 131-136. doi: 10.1016/j.conb.2008.06.002

Eagleman, D. M., and Holcombe, A. O. (2002). Causality and the perception of time. Trends Cogn. Sci. 6, 323-325. doi: 10.1016/S1364-6613(02) $01945-9$

Eagleman, D. M., Tse, P. U., Buonomano, D., Janssen, P., Nobre, A. C., and Holcombe, A. O. (2005). Time and the brain: how subjective time relates to neural time. J. Neurosci. 25, 10369-10371. doi: 10.1523/JNEUROSCI.348705.2005

Efron, B., and Tibshirani, R. J. (1993). "An introduction to the bootstrap," in Refrigeration and Air Conditioning, (Vol. 57) eds D. R. Cox, D. V. Hinkley, N. Reid, D. B. Rubin and B. W. Silverman (Boca Raton, FL: Chapman \& Hall), 436

Erim, Z., Beg, M. F., Burke, D. T., and de Luca, C. J. (1999). Effects of aging on motor-unit control properties. J. Neurophysiol. 82, 2081-2091.

Finney, D. J. (1947). Probit Analysis: A Statistical Treatment of the Sigmoid. Cambridge, MA: Cambridge University Press.

Gelfand, S. A., Hoffman, S., Waltzman, S. B., and Piper, N. (1980). Dichotic CV recognition at various interaural temporal onset asynchronies: effect of age. J. Acoust. Soc. Am. 68, 1258-1261. doi: 10.1121/1.385117

Haggard, P., and Chambon, V. (2012). Sense of agency. Curr. Biol. 22, R390-R392. doi: 10.1016/j.cub.2012.02.040

Heron, J., Hanson, J. V. M., and Whitaker, D. (2009). Effect before cause: supramodal recalibration of sensorimotor timing. PLoS One 4:e7681. doi: 10.1371/journal.pone.0007681

Heron, J., Roach, N. W., Whitaker, D., and Hanson, J. V. M. (2010). Attention regulates the plasticity of multisensory timing. Eur. J. Neurosci. 31, 1755-1762. doi: $10.1111 / j .1460-9568.2010 .07194 . x$

Keetels, M., and Vroomen, J. (2012). Exposure to delayed visual feedback of the hand changes motor-sensory synchrony perception. Exp. Brain Res. 219, 431-440. doi: 10.1007/s00221-012-3081-0
Laurienti, P. J., Burdette, J. H., Maldjian, J. A., and Wallace, M. T. (2006). Enhanced multisensory integration in older adults. Neurobiol. Aging 27, 1155-1163. doi: 10.1016/j.neurobiolaging.2005.05.024

Liu, X. Z., and Yan, D. (2007). Ageing and hearing loss. J. Pathol. 211, 188-197. doi: $10.1002 /$ path. 2102

Madden, D. J. (2007). Aging and visual attention. Curr. Dir. Psychol. Sci. 16, 70-74. doi: 10.1111/j.1467-8721.2007.00478.x

Maki, B. E., and Mcllroy, W. E. (1996). Postural control in the older adult. Clin. Geriatr. Med. 12, 635-658.

McDowd, J. M., and Oseas-Kreger, D. M. (1991). Aging, inhibitory processes, and negative priming. J. Gerontol. 46, P340-P345. doi: 10.1093/geronj/46. 6.p340

Moore, J. W., and Fletcher, P. C. (2012). Sense of agency in health and disease: a review of cue integration approaches. Conscious. Cogn. 21, 59-68. doi: $10.1016 /$ j.concog.2011.08.010

Moore, J. W., Lagnado, D., Deal, D. C., and Haggard, P. (2009). Feelings of control: contingency determines experience of action. Cognition 110, 279-283. doi: 10.1016/j.cognition.2008.11.006

Mozolic, J. L., Hugenschmidt, C. E., Peiffer, A. M., and Laurienti, P. J. (2012). "Multisensory integration and aging," in The Neural Bases of Multisensory Processes, eds M. Murray and M. T. Wallace (Boca Raton, FL: CRC Press), 381-395.

Ostroff, J. M., McDonald, K. L., Schneider, B. A., and Alain, C. (2003). Aging and the processing of sound duration in human auditory cortex. Hear. Res. 181, 1-7. doi: 10.1016/s0378-5955(03)00113-8

Raz, N., Lindenberger, U., Rodrigue, K. M., Kennedy, K. M., Head, D., Williamson, A., et al. (2005). Regional brain changes in aging healthy adults: general trends, individual differences and modifiers. Cereb. Cortex 15, 1676-1689. doi: 10.1093/cercor/bhi044

Robin, D. A., and Royer, F. L. (1989). Age-related changes in auditory temporal processing. Psychol. Aging 4, 144-149. doi: 10.1037/0882-7974. 4.2.144

Schmolesky, M. T., Wang, Y., Pu, M., and Leventhal, A. G. (2000). Degradation of stimulus selectivity of visual cortical cells in senescent rhesus monkeys. Nat. Neurosci. 3, 384-390. doi: 10.1038/73957

Seidler, R. D., Bernard, J. A., Burutolu, T. B., Fling, B. W., Gordon, M. T., Gwin, J. T., et al. (2010). Motor control and aging: links to age-related brain structural, functional, and biochemical effects. Neurosci. Biobehav. Rev. 34, 721-733. doi: 10.1016/j.neubiorev.2009.10.005

Setti, A., Finnigan, S., Sobolewski, R., McLaren, L., Robertson, I. H., Reilly, R. B., et al. (2011). Audiovisual temporal discrimination is less efficient with aging: an event-related potential study. Neuroreport 22, 554-558. doi: 10.1097/WNR. 0b013e328348c731

Shaffer, S. W., and Harrison, A. L. (2007). Aging of the somatosensory system: a translational perspective. Phys. Ther. 87, 193-207. doi: 10.2522/ptj.20060083

Spear, P. D. (1993). Neural bases of visual deficits during aging. Vision Res. 33, 2589-2609. doi: 10.1016/0042-6989(93)90218-1

Spear, P. D., Moore, R. J., Kim, C. B., Xue, J. T., and Tumosa, N. (1994). Effects of aging on the primate visual system: spatial and temporal processing by lateral geniculate neurons in young adult and old rhesus monkeys. J. Neurophysiol. 72, $402-420$.

Stetson, C., Cui, X., Montague, P. R., and Eagleman, D. M. (2006). Motor-sensory recalibration leads to an illusory reversal of action and sensation. Neuron 51, 651-659. doi: 10.1016/j.neuron.2006.08.006

Stevenson, R. A., Baum, S. H., Krueger, J., Newhouse, P. A., and Wallace, M. T. (2017). Links between temporal acuity and multisensory integration across life span. J. Exp. Psychol. Hum. Percept. Perform. doi: 10.1037/xhp0000424 [Epub ahead of print].

Sturr, J. F., Zhang, L., Taub, H. A., Hannon, D. J., and Jackowski, M. M. (1997). Psychophysical evidence for losses in rod sensitivity in the aging visual system. Vision Res. 37, 475-481. doi: 10.1016/s0042-6989(96)00196-4

Sugano, Y., Keetels, M., and Vroomen, J. (2010). Adaptation to motor-visual and motor-auditory temporal lags transfer across modalities. Exp. Brain Res. 201, 393-399. doi: 10.1007/s00221-009-2047-3

Tinetti, M. E., Speechley, M., and Ginter, S. F. (1988). Risk factors for falls among elderly persons living in the community. N. Engl. J. Med. 319, 1701-1707. doi: $10.1056 /$ nejm198812293192604 
Turgeon, M., and Wing, A. M. (2012). Late onset of age-related difference in unpaced tapping with no age-related difference in phase-shift error detection and correction. Psychol. Aging 27, 1152-1163. doi: 10.1037/a0029925

Turgeon, M., Wing, A. M., and Taylor, L. W. (2011). Timing and aging: slowing of fastest regular tapping rate with preserved timing error detection and correction. Psychol. Aging 26, 150-161. doi: 10.1037/a0020606

Vercillo, T., Burr, D., Sandini, G., and Gori, M. (2014). Children do not recalibrate motor-sensory temporal order after exposure to delayed sensory feedback. Dev. Sci. 18, 703-712. doi: 10.1111/desc. 12247

Vesco, K. K., Bone, R. C., Ryan, J. C., and Polich, J. (1993). P300 in young and elderly subjects: auditory frequency and intensity effects. Electroencephalogr. Clin. Neurophysiol. 88, 302-308. doi: 10.1016/0168-5597(93)90054-s

Warren, L. R., Wagener, J. W., and Herman, G. E. (1978). Binaural analysis in the aging auditory system. J. Gerontol. 33, 731-736. doi: 10.1093/geronj/33. 5.731
Wolpe, N., Ingram, J. N., Tsvetanov, K. A., Geerligs, L., Kievit, R. A., Henson, R. N., et al. (2016). Ageing increases reliance on sensorimotor prediction through structural and functional differences in frontostriatal circuits. Nat. Commun. 7:13034. doi: $10.1038 /$ ncomms 13034

Conflict of Interest Statement: The authors declare that the research was conducted in the absence of any commercial or financial relationships that could be construed as a potential conflict of interest.

Copyright (c) 2017 Vercillo, Carrasco and Jiang. This is an open-access article distributed under the terms of the Creative Commons Attribution License (CC BY). The use, distribution or reproduction in other forums is permitted, provided the original author(s) or licensor are credited and that the original publication in this journal is cited, in accordance with accepted academic practice. No use, distribution or reproduction is permitted which does not comply with these terms. 